\title{
Steinzeitliche Wirtschaftsformen
}

\author{
Von A. Schliz
}

Das Bild, welches die Besiedlung Mitteleuropas in der jüngeren Steinzeit bietet, ist bekanntlich ein recht vielgestaltiges. Dass es Völker verschiedener $\mathrm{R}$ a s s e n waren, welche diesen Boden teils nebeneinander, teils nacheinander bewohnten, hat die Schädeluntersuchung der hinterlassenen Skelette, dass innerhalb dieser Siedlungsgebiete verschiedene scharf geprägte $K$ u l t u r e n bestanden, hat die Untersuchung und Vergleichung der im Boden zurückgebliebenen Reste ihrer Geräte, Waffen und Töpferarbeiten nachreisen können. Die eingehende Untersuchung ihrer Wolunstätten auf ihre Lage, Gruppierung. Form und ihren Kulturinhalt hat aber weiter ergeben, dass auch die Wirtsch a $\mathrm{t}$ s f or m der einzelnen Stämme eine grossenteils recht verschiedene war. Die Unterschiede treten hervor durch den Vergleich der Haus- und Jagdtiere, der Gebrauchsgeräte, Töpferware, Waffen, Bekleidung, Wohn- und Begräbnisform, aus denen wir auf die wirtschaftliche Stellung der Berrohner unseres Bodens schliessen können.

Anch auf unsere prähistorischen Zustände können wir die von den Ethnologen geprägten Ausdrücke für die verschiedenen wirtschaftlichen Stufen anwenden. Einzelne dieser Volksstämme befanden sich sichtlich mirtschaftlich auf der Sammelstufe mit Jagd; Fischfang und Sammeln wildwachsender Bodenfrüchte als wirtschaftlicher Grundlage, andere auf der $\mathrm{H} \mathrm{ackba} \mathrm{u-oder} \mathrm{Pflanzenbaustufe,} \mathrm{bei} \mathrm{der} \mathrm{die} \mathrm{Nahrung}$ durch planmässigen Anbau bestimmter Bodenfrüchte auf bequem gelegenem Gelände gewonnen und durch die Ergebnisse von Jagd und beschränkter Hanstierhaltung ergäinzt wird; andere endlich haben eine auf Pflugbau und Züchten grosser Viehherden aufgebaute Hoch-oder Vollkultur. Von vornberein müssen wir uns aber der Vorstellung entschlagen, als ob das Verharren auf der wirtschaftlichen Form der Sammelstufe einen niedrigeren, "primitiveren" Stand menschlicher Kultur im ganzen in sich schliesse und dass als natürliche Stufenfolge der menschlichen Kulturentwicklung stets ein Fortschreiten der wirtschaftlichen Entwicklung von der Sammelstufe zum Hackban und ron da zur Vollkultur zu beobachten wäre; die wirtschaftliche Stufe der einzelnen Volksstämme richtet sich vielmehr in der Hauptsache nach der Natur des Bodens, den sie bewohnen, und wohl auch nach den aus den geologischen Terhältnissen ihres Ursprungslandes errachsenen und traditionell weiter gepflegten Lebensformen. Dabei findet jedoch in einzelnen Fällen Ubernahme von Kulturgut aus der einen Stufe in die andere statt. Ob solche Mischkulturen dann auf dem Aufgehen eines bestimmten, bisher auf anderer Stufe befindlich gewesenen Volkselementes in einer bis dahin in sich abgeschlossenen Stammesgeneinschaft beruhen, ist meist noch eine offene Frage.

Bei der grossen Ausdehnung und der kulturellen Vielgestaltigkeit des mitteleuropäischen Siedlungsgebietes soll als Grundlage dieser Unter- 
suchung ein Ausschnitt aus demselben, das nördliche Voralpengebiet bis zum

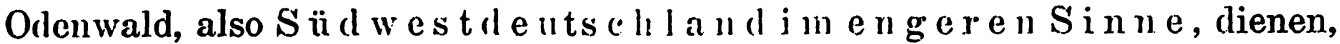
ein durch die Riegel des Schwarzwaldes, Odenwaldes und der dentschen Mittelgebirge natürlich begrenztes und abgescllossenes Siedlungsgebiet, welches dazu noch durch stufenweisen Wechsel der Bodenformation die Grundlage für verschiedene Wirtschaftsformen an sich schon bietet. Für Kultur- und Bevölkerungszuflüsse aus den Nachbargebieten standen in der Hauptsache drei Wege offen, von denen der eine donauaufwärts, der zweite neckaraufwärts, der dritte vom Obermain her meist auf schmalen Bahnen ins Innere dieses Gebietes führt, und wirklich ist auch das Kulturgut, welches nicht seinen Ursprung in Lande selbst hat, ohne weiteres als auf diesen drei Wegen direkt aus bestimmten Kulturzentren der Nachbarläncler eingeführt zu erkennen.

Deutlich unterscheiden sich hier drei grosse Kulturkreise, teils nach ihren keramischen Besonderheiten, teils nach ihrer Siedlungsform benannt, der schnurkeramische, bandkeramische und Pfahlbaukulturkreis, in welche besondere Unterabteilungen sich einfügen. Unter diesen letzteren spielt die anfangs als Kultur- und später als Bevölkerungszuwanderung aus Mitteldeutschland eindringende $\mathbf{R}$ ös s e n er Kultur eine besondere .Rolle.

Rassenanthropologisch gehören die Völker des schnurkeramischen Kulturkreises und von dem, was wir als Bandkeramikkultur zusammenfassen, die der Hinkelstein-, Grossgartacher- und Donau-Kultur der mittelländischen Völkerfamilie, die der Rössener Kultur der nordländischen und die der Pfahlbaukultur der alpenländischen Rasse an, wie sich aus den Schädelformen bestimmt nachweisen lässt.

Von diesen Völkern müssen wir als Ureiüwohner Südwestdeutschlands mittelländische Stämme und die Pfahlbaurasse betrachten, welche lange Zeit durch die Grenze des Albabsturzes getrennt selbständig ihre Kulturen entwickelten. Das Bodenseegebiet und die schwäbische Hochebene gehörten zum Wirtschaftsgebiet der Pfahlbäustämme, die Lössflächen des Unterlandes bis zum Odenwald den Völkern der Hinkelstein- und donauländischen Bandkeramikkultur an. In dieses unterländische Gebiet, dessen Kultur sich in stetem Zusammenhang mit der der oberen Donau weitei entwickelt hat, wanderten aus Mitteldeutschland unsesshafte Stämme des schnurkeramischen Kulturkreises und nordländische Träger der Rössener Kultur ein und wirkten befruchtend auf die alte Hinkelstein- und Donaukultur ein. Anfangs werden die Rössener Formen wohl Kulturwanderung gewesen sein, später aber sehen wir echte Siedlungen dieser Stämme selbst über die schwäbische Hochebene und das Rheintal bis zum Bodensee vordringen. Am Ende der Steinzeit verschwinden die bandkeramischen Siedler, und Südwestdeutschland wird dicht mit Höhensiedlungen alpiner Stämme beI setzt, in denen wir Pfahlbau-, Rössener und schnurkeramische Elemente zu einer Mischkultur, der Mi c h e ls b e r g e r, vereint sehen, bereits durchsetzt mit kupferzeitlichen Formen. Hier, wie in den Ursprungsstiitten der aus Rössener und Pfahlbauelementen zusammengesetzten Schussen- 
r i e d e r Mischkultur ist jede Spur der donauländischen Bandkeramik verschwunden; nur spärliche Ornamente aus dem Kreis der kupferzeitlichen Mondseekeramik halten die Verbindung aufrecht.

Von den drei grossen geschlossenen Kulturen ist die s c h n u r k e r a m i s c h e K u l t ur auf südwestlentschem Boden trotz ilhrer primitiven Gebrauchskeramik keine neolithische Frühlsultur. In 'Thüring'en alteinheimisch, treten diese Stämme zur Zeit der bandkeramischen Hochkultur in Südwestdeutschland vom Main her auf und überdauern sie bis zur Kupferzeit. Nach dem Wèg, den ihr Erscheinen bėzeichnet, und der Lage ihrer: Grabliügel müssen wir für ilıre Wanderung deu Landweg annehmen, und vielleicht gehen die aus Jagdpfaden entstandenen Höhenwege Südwestdeutschlands bis anf diese Zeit zurück, während die bandkeramischen Siedlungen durchweg dem Laufe der Flüsse folgen. Wahrscheinlich sind diese Jägerstämme gleichzeitig mit der Rössener Südwanderung ins Land gekommen. Durch ihre Wirtschaftsform hatten sie stets neben den Ackerbaustämmen Platz; sie werden also in Mitteldeutschland ebenso wie später im Süden neben bandkeramischen und Rössener: Stämmen ausgedehnte Jagdgebiete im Besitz gehabt haben. Wenn wir von dem späteren Übergang. ihrer Kultur auf die Pfahlbauten der Westschweiz absehen, besitzen wir in Südwestdeutschland als Hinterlasseuschaft von ihnen lediglich Gräher, anfangs Steinkisten, dann Schachtgräber unter aufgeschütteten Hügeln mit in Hockerstellung liegenden und gestreckten Skeletten, und schliesslich Leichenbrand im Hügelgrab und einfachen Gruben. Diese Gräber enthalten keine Geräte des täglichen Lebens, sondern ausschliesslich Waffen: Fenersteinlanzen, trapezförmige Wurfbeile mit rechtwinkligem Querschnitt. Feuersteinmesser, sorgfältig geschliffene und polierte durchlochte Beilhänımer, aber keine Pfeile. Dazu kommen neben einer plumpen, wesentlich Kumpen, Becher und Töpfe aufweisenden Gebrauchskeramik die feinen. durch Eindrücken von Schnüren in einen feingeschlämmten roten oder gelben Tonüberzug hergestellten geometrischen Ornamente auf Bechern. Krügen und Amphoren, von denen die ganze Kultur ihren Namen hat. Diese in horizontalen Reihen die Gefässe umziehenden geometrischen Ornamente stellen eine eigentliche Kunstblüte dar, und ich bin auch hente noch der Ansicht, dass die eigentümliche Halsdekoration der Gefäisse des Grossgartacher Stils in horizontalen Stichreihen und die Schnurimitationen auf handkeramischen Linienbandgefässen von ihnen übernommen worden șind. Ton Tierknochen haben sich bis jetzt nur Jagdtierknochen gefunden, ausserlem aber im Grabhügel vom Hebenkies bei Wiesbaden das Pferd. Trotzdem liese Gräber die grosse steinzeitliche Niederlassung von Grossgartach in weiteun Kreis umgeben und in einzelnen Wolmstätten dieser alle Stufen ler Bandkeramik, Hinkelstein-, donauländischen, Grossgartacher und Rössener Keramik grossenteils in denselben Wohnstätten aufweisenclen Niederlassung sich Trapezheile mit rechteckigem Querschnitt gefunden haben, ist nicht eine Wohnung zum Vorschein gekommen, die wir der schnurkeramisichen Kultur speziell als solcher zuschreiben könnten. Steinwaffenbearbcitungsstätten, wie in Mitteldentschland, oder offene Lagerplätzc mit Tier- 
knochen deckt vielleicht noch der Waldbestand, in dem die Grabhügel liegen, zu; in ganzen ist für diese Wirtschaftsform dieselbe Stufe anzunehrnın wie die der ostbaltischen Neolithiker, welche im Lagerplatz von $\mathrm{R}$ i n n e k a l n nur Jagdtierknochen und die Reste einer kleinen Hunderasse hinterlasesn haben. Diese Stämme sind sichtlich reine Jägerstämme, jedoch mit entwickelter Industrie in Waffen und Töpferei, gewesen; ihre Wohnform war das Zelt und ihre Wirtschaftsform die S a m melst u f e.

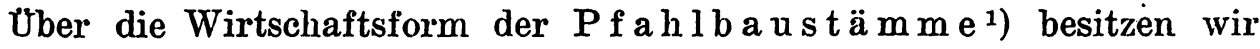
wesentlich positivere Kenntnisse. Wir müssen aber hier zwei für sich entwickelte Kulturen unterscheiden, wenn sie auch ursprünglich derselben Bevölkerung angehörten. Die Bewohner der nordschweizerischen und Bodenseepfahlbauten wohnen in Dauersiedlungen mit fest gebauten, häufig zweigelassigen Viereckhäusern mit steilen Wänden und freiliegendem Dach. A uch jetzt noch bildet Früchtesammeln und Jagdbeute die Hauptnahrungsquelle. Äpfel, Pflaumen, Himbeeren, Erdbeeren und Wassernüsse entstammten sicher wildwachsenden Fruchtträgern, aber als erster absichtlicher Pflanzenanbau erscheint Weizen und Gerste. Edelhirsch, Reh, zwei Arten von Wildschwein, Elentier, Ur, Wisent, Bär, Biber, Fuchs, Dachs, Igel, Schwan, Wildente, Haselhuhn und Wildtaube werden erlegt und gegessen, Barsch, Karpfen, Hecht und Lachs gefangen, aber als Haustiere erscheinen die Ziege, das gezähmte Torfschwein und als Milchvieh der bos brachyceros. Erst in den späteren Pfahlbauten erscheint der schwere bos taurus primigenius, wie auch das seltene Pferd. Als Begleiter dient ein kleiner, wachtelhundähnlicher Jagdhund. Die Töpferei liefert grosse und kleine Gebrauchsgefässe, gleich vorzüglich in Material; Brand und Technik. Gross ist die Zahl der aus Holz, Knochen und Horn technisch vorzüglich und zweckmässig gearbeiteten Hausgeräte, die uns der Seeschlamm erhalten hat. Von Steingeräten überwiegt die Hacke, „das Pfahlbaubeil“, grossenteils zur Fassung in Hirschgeweih und Holz eingerichtet, weitaus die übrigen Formen. Dieses Pfahlbaubeil diente, wie die Hammeraxt, ebenso als Waffe wie als Arbeitsgerät...An die. Seite tritt ihm die Hacke aus Hirschgéweih. Keines dieser Werkzeuge ist als Pflugschar verwendbar. Die ganze Bodenwirtschaft befindet sich auf der $\mathrm{H} \mathrm{a} \mathrm{ck} \mathrm{b}$ a u s t u f e. Ausserordentlich zahlreich sind die Pfeilspitzen aus Silex; der Bogen war sichtlich die Hauptwaffe bei Jagd und Krieg. Zur Bekleidung dienten neben Tierhäuten geflochtene und gewobene Stoffe aus Pflanzenfasern: Für die Leichenbestattung ist nirgends der Leichenbrand nachweisbar. Die Begräbnisform ist bei diesen Wassersiedlungen schwer nachweisbar; wenn wir aber die bei den Landsiedlungen, die derselben Bevölkerung entstammten, übliche Sitte heranziehen, so haben wir Erdbegräbnis in tiefen Grubeu als Hocker auf dem Michelsberg und zweistufige Bestattung, Beisetzung des Schädels in einer Vorratsgrube, auf dem Goldberg. Wenn wir die Kennzeichen des von W. Foy und Gräbner aufgestellten Begriffs der (melanesischen) Bog e n -

1) Rütimeyer, Faụna der Pfahlbauten der Schweiz. - v. Tröltsch, Die Pfahlbauten des Bodenseegebiets. - Leiner, Das Pfahlbauwesen am Bodensee. - 
kultur zusammenfassen: Hackbau, Pfahlhäuser, Rechteckhütten mit Giebeldach, Bogen und Pfeil, Knochendolch, Erdbegräbnis als Hocker, zweistufige Bestattung; geflochtener Lendengürtel, so fehlt für unsere Pfahlbaukultur kein Kennzeichen, um sie nicht ebenfalls „Bogenkultur“ zu nennen. Es felılt nur noch der Totemismus, über dessen Vorhandensein wir freilich nichts wissen können.

Eng angeschlossen, aber in selbständiger Entwicklung, tritt der Pfahlbaukultur die Michelsberger Kultur an die Seite, über welche wir in ihrem südwestdeutschen Teil durch die Ausgrabungen A. B on nets auf dem Michelsberg und G. B e r s u s auf dem Goldberg genau und zuverlässig unterrichtet sind. In ihrer Blüte war sie im nördlichen Voralpengebiet so weit verbreitet, dass nahezu jede freie Bergkuppe eine ihrer Höh e n s i ed l u n g e n trägt. In diesen Siedlungen sehen wir die Wirtschaftsform der $\mathrm{Hackbaustufe}$ weiter entwickelt. Bodenhacken und richtige Spaten werden vorzugsweise aus Knochen und Hirschgeweih angefertigt; ganz hervorragend ist neben der Hirschgeweihbearbeitung die Steinindustrie in geschlagenem Silex, der namentlich zur Herstellung zahlreicher Pfeilspitzen dient, so dass wir auch hier von „Bogenkultur“ reden können. Die Jagd steht immer noch im Vordergrund des wirtschaftlichen Lebens, aber es erscheint jetzt neben dem Torfrind bos taurus und Schaf als Weidevieh. Die Wohnform ist auch hier das Rechteckhaus mit festen, steilen Wänden; aber der erhöhte Hackbau fügt runde, als Trichtergruben angelegte Speicher mit Zeltdach für die Fruchtvorräte hinzu. Auf dem Goldberg sind zwei Formen von Rechteckhäusern hervorgetreten, Pfostenhäuser , mit ebenem Innern und Grubenhäuser mit durch Streben verstärkten Wänden und Kellergeschossen. In letzterer Bauweise ist wohl fremder Einfluss zum Ausdruck gekommen. Aber auch in der Töpferei, den Steingeräten und Waffen tritt ein solcher Einfluss jetzt deutlich hervor. Die Tongefässe bekommen elegante Formen und nach aussen geschwungene Ränder, und Material, Brand und Glättung sind technisch so durchgebildet, dass glatte Scherben aus der Steinzeitschicht des Goldberges von denen der an der Oberfläche liegenden Hallstattbesiedlung kaum zu unterscheiden sind. Zahlreich erscheinen jetzt fasettierte durchlochte Beilhämmer und spitznackige dreieckige Flachbeile aus den späten Kulturkreisen der Schnurkeramik und Dolmenkultur.

Am zahlreichsten sind auf dem Goldberg die Zuflüsse aus der Rös s e ner Kultur, welche schon auf dem Michelsberg hervorgetreten waren. Diese von Zuwanderern nordländischer Rasse nach Südwestdeutschland hereingetragene Kultur, entstanden aus der Einwirkung bandkeramischer Elewente auf die nordische tiefstichornamentierte Formengebung, gelangt hier bis an den Bodensee und geht auf dem Albplateau eine neue Verbindung mit der alteinheimischen Pfahlbaukultur ein, die ihren Ausdruck in einem nenen Typus, dem Sch ussenrieder, findet. Was sich hier an Schuhleistenkeilen, dem bandkeramischen Universalwerkzeug, findet, ist Rössener Ursprungs. Diese Bewegung ist aber als eine späte anzusehen, wie das Vojkomunen des Rössener Kulturinventars in ungetrennter Mischung mit de॥ Resten schnurkeramischer Dolmen- und Mondseekultur in denselben Wohn 
stätten und Schichten auf dem Goldberg ausweist. Neben dem Schuhleistenkeil führt aber auch diese Bevölkerung die Hirschhornhacke, und es ist walurscheinlich, dass sie sich wirtschaftlich ebenfalls auf der $\mathrm{Hack}-$ b a us $t$ u f e befand.

Inı württembergischen Unterland finden sich die Rössener Wohnstätten eingestrent in das ba nd keram is che Siedlungsgebiet mit d o n a u lä n discher und Grossgartacher Kultur. Das gleichyeitige Bestehen clieser beiden Stilformen in denselben Wohnstätten ist einwaindfrei nachgewiesen und auch von Mainz her offiziell anerkannt. Diese keramische Entwicklung dïrfte sich in folgender Weise vollzogen haben: Der Ausgangspunkt der ganzen Stilbewegung, die wir donauländische Bandlieramik neunen, ist ein umfangreiches, mit reichen I.össflächen ausgestattetes Gebiet, das Niederösterreich, das bayerische Donaugebiet, Mähren und Böhmen umfasst. Das Grundprinzip ist ursprünglich die Dekoration einer glatten, möglichst unkomplizierten Gefässfläche, des Kugeltopfes, die hierzu zeichnerisch in gleichniässige Segmente eingeteilt wurde. Die Zeichnungen waren linear teils in einfachen, teils in unterbrochenen Linien ausgefuihrt. Als diese Dekorationsweise bei den sesshaft gewordenen südwestdeutschen und rheinischen Stämmen mittelländischer Rasse aufkam, war bereits die Neig'ung vorhanden, bestimmte Gefässteile, namentlich den Hals, durch friesartig umlaufende Bänder, sei es auch nur durch Fingernageleindrücke, hervorzuheben und die linearen Muster, in erster Linie die Zickzacklinien, teils einfach, teils als parallele Gruppen in Winkelstellungen anzuordnen. So entstand der südwestdeutsche Hinkelsteinstil, während im Ursprungsgebiet die Entwicklung von Spirale und Mäander der Winkelliniendekoration parallel ging. Wenn in Böhmen wirklich die „Stichbandkeramik“ mit ihren Winkelstellungen erst die zweite Stufe bildet ${ }^{1}$ ), so gehört eben Böhmen nicht zum Ursprungsgebiet der Bandkeramik, wenn sie auch clort in der parallelen Ausbildung des ganzen Formenschatzes der Stichband- und linearen Winkel-, Mäander- und Volutenornamentierung eine besonders hohe Kunstblüte entwickelt hat, an der die mitteldeutschen Lössgebiete vollen Anteil nehmen ${ }^{2}$. Als die Linienbandornamentik mit ihren Winkel-, Spiral- und Mäandermustern aus dem Donaugebiet ihren Einzug in das engere Südwestdeutschland hielt, war dort der Hinkelsteinstil bereits in der Umbildung zum Grossgartacher Stil begriffen. An dieser Umbildung haben verschiedene Faktoren, die Rössener Dekorationsweise bestimmter, jetzt auch in der Profilierung scharf hervorgehobener Gefässteile durch Stichreihenmuster, die mit jener eingezogene Schnurkeramik in der horizontalen Anordnung der Bänder und die donauländische Lineardekoration mit Bogenstellungen und Freiornamenten, Anteil gehabt: In Mitteldeutschland, am Main und Mittelrhein führte dann das Verlassen des strengen geometrischen Prin-

1) Emanuel Simek, Grundzüge der Vorgeschichte Böhmens. Wiener Prühist. Zeitschrift Band I, Heft 1, 2. Bemerkenswert ist Abb. 2 mit der Bezeichnung „Spiralmäanderkeramik $^{\alpha}$ auf der nur Winkelstellungen mit Füllstücken = Strichen zu sehen sind.

2) Siehe auch Bärthold-Halberstadt, Korrespondenzblatt der Deutschen Anthropol. Gesellschaft XLV. Nr. 5. 
zips in der zeichnerischen Einteilung der Gefässwand zun reinen Freiornainent, für welches die gesamte Gefässwand als Zeichnungsgrund für gebogene und gebrochene Einzelspiralen benutzt wird, wie es der Flomborner Stil so prägnant zum Ausdruck bringt. In unser Gebiet sind nur wenige in dieser Weise dekorierte Gefässe neckaraụfuärts gewandert, vergesellschaftet mit Gefäissen der vom Main bis nach Belgien verloreiteten Eichelsbacher Dekorationsweise mit ihren durch gekreuzte Strichlagen gefüllten Schlangenbändern. Wir haben hier eine Parallele zu den netzartig schrafficrten Hängebändern der entwickelten Grossgartacher Dekoration. Rassenanthropologisch hat sich die Bevölkerung mit Grossgartacher Kultur durch die Skelette vou Grossgartach, Friedberg, Lingolsheim und Niederingelheim als der grossen mittelländischen Völkerfamilie angehörig erwiesen, die grosswiichsige Stämme, welche wir den Lenggeltypus neunen können, und kleinwüchsige, denen der bölımische Typus angehört, umfasst. Die W i r t sch af tsform dieser. Stämme ist eine ausgesprochene Hoch- und rollk ultur, Pflugbau des Ackers und Züchten grosser Viehherden als Zng- und Schlachttiere. Es kommen steinerne Pflugscharen bis zu $40 \mathrm{~cm}$ Iänge ror, und in Grossgartach crscheint ein starker Hakenpflug aus der Augsprosse eines mächtigen Hirschgeweihs. Die ganze $S$ t e in ind u s trie, der Schuhleistenkeil und das halbseitig gewölbte Flachbeil dient gewerblichen Zwecken; Pfeile werden sehr selten, Isanzen fehlen ganz. Die erhaltenen $\mathrm{B}$ e in a r tef a k te und Handwerksgeräte, Glätter, Schaber, Pfriemen und Nadeln dienten zur Bearbeitung des Leders, die vorhandenen Schiff̈chen schwerlich zur Weberei, sondern zum Stricken von Netzen und Herstellung grober Geflecbte. Die Zahl der Spinnwirtel ist so gering und ihre Form so kunstlos, dass an die Herstellung von eigentlichen Gespinsten kaum gredacht werden kamn. Die Kleidung war im Winter wohl Pelz und Lederschurz oder Beinkleid, den Malereien auf Hauswänden und Gefässen nach wohl auch bunt verziert, im Sommer wird man den Oberkörper nackt getragen haben. Die Begräbnisform ist teils Beisetzung im Flachgrab, wie jn Lingolsheim, Niederingelheim und Sontheim bei Heilbronn, auch in Wohnstätten, wie in Grossgartach und Friedberg, teils Leichenbrand, wie im Naintal. Es ist noch nicht aufgeklärt, wo die Menge von Leichen aus der solange bestandenen Niederlassung von Grossgartach hingekommen ist. Bei der systematischen Durchforschung des Bodens und dem Bestehen einer Reihe von Kies- und Lehmgruben hätten doch irgendwo Gräberfelder zum Vorschein kommen müssen. Als eigentliche W a f f e n kennen wir nur die wenigen Pfeile, die wohl vorwiegend zur Jagd dienten. fiejagt wurde hauptsächlich Hirsch und Reh; das Wildschwein fehlt. Jler zahlreich vorhandene Ur und der Biher wurden wohl nur in Fallen gefangrn. Von $\mathrm{H}$ a ustieren überwiegt die schwere bos taurus-Rasse jetzt wejt das kurzhörnige Milchvieh; zahlreich wird ein grosses hochbeiniges Hausschwein gezüchtet. Schafe wurden zahlreich, wahrscheinlich in Herd(ni, Zjegen einzeln in den nicht selten an kleine Wohnungen angebauten Staillon gehalten; vom Pferd findet sich in Grossgartach ein einziger Hengst\%ahı, der nirht örtlicher Zucht \%u entstammen braucht. 
Die W o h $\mathrm{n}$ f or $\mathrm{m}$ ist bei dieser Hochkultur nicht einheitlich; sie richtet sich nach der Art des wirtschaftlichen Betriebes. In der grossen, besonders eingehend untersuchten Niederlassung von Grossgartach-Frankenbach herrscht der Gehöftebau mit Wohnhaus und zugehörigen, für sich stehenden Ställen und Scheunen vor. Die Grossgartacher rechteckigen, zweigelassigen Wohnhäuser mit festen, aus Flechtwerk und Lehmpatzen aufgefülırten, innen glatt verputzten und bemalten Wänden sind bekannt. Wer an der Richtigkeit der Bonnetschen Grabungen noch zweifelt, kann die Wandkonstruktion an dem massenhaften, im Heilbronner Museum aufbewahrten flachen Wandverputz studieren. Unverkennbar ist die Parallele mit dem Pfahlbauhaus in seiner Schussenrieder Form. Diese Wohnhäuser sind auch gleich konstruiert, einerlei, ob ihr Inneres mehr donauländische oder Grossgartacher Keramik enthält. Noch deutlicher tritt der Einfluss des Pfahlbaukulturkreises bei den von G. B e r s u bei den Grabungen im Kastell R o t $t$ w'e il aufgedeckten neolithischen Häusern mit Linienbandkeramik hervor. Es sind das ,ausgedehnte Grubenwohnungen mit annähernd geradliniger Begrenzung nach aussen und tiefen Mulden im Innern in ungewöhnlich klarer Begrenzung. Reihen von Pfostenlöchern mit bis $40 \mathrm{~cm}$ Durchmesser lassen auf grosse Pfostenbauten mit geradliniger Wandung schliessen “1). Von den durch tiefschwarzen, schollig brechenden, scherbenarmen Grubeninhalt gekennzeichneten Grossgartacher Ställen hatten nur die grossen, von den $S$ che $\mathrm{n}$ en und $\mathrm{S}$ tad eln beinahe keine festgebaute, verputzte Wände. Letztere liegen oberflächlich und sind meist schon von weitem als dunklere Flecke im helleren Ackerboden kenntlich. Die äussere Begrenzung derselben tritt meist erst nach Ausleerung der 'ganzen Grube und auch dann noch selten scharf hervor. Wir.haben deshalb auch keinen dieser Grundrisse in unsere Publikationen aufgenommen, doch konnten wir 1913 einen solchen Stadel ohne festgebaute Wände, bei dem der rechteckige Umriss und die zweigelassige Inneneinteilung erhalten geblieben war, einer Anzahl von Forschern vorführen. Das Ergebnis ist durch G. B e r s u und mich im „Römisch-Germanischen Korrespondenzblatt" veröffentlicht. Beinahe alle diese Vorratshäuser enthalten im Innern einen rechteckigen Keller oder eine tiefe, runde Vorratsgrube. Da, wo der oberflächliche Hüttenbau verschwunden ist, ist häufig die Grube allein übrig geblieben. Nach Analogie der Wohnhäuser haben wir für alle rechteckigen Oberbau angenommen; es ist aber nicht ausgeschlossen, dass solche runde, tiefe Gruben für sich angelegt und mit einem Zeltdach überdeckt wurden. Bestimmt nachweisen haben wir dies aber nie können, da keine Pfahlumgrenzungen sicher hervortraten.

Neben der Frage der Pfostenumgrenzungen haben uns aber die mainländischen Ergebnisse noch zur Untersuchung der grossen Flächen schwarzen Bodens geführt, die, in den Aussengebieten der Grossgartacher Niederlassung liegend, von uns bisher nach dem Ergebnis der Versuchsgräben als

- Viehhöfe angesehen worden waren. Der Hauptgrund der bisherigen Vernachlässigung lag darin, dass sie bei der geringen Ackerbreite der Gross-

1) Fundberichte aus Schwahen. XXI. Jahrg. 1913, S. 78. 
gartaclicr Gewanne alle durch mehrere Ackerbreiten durchgehen, von denen regelmässig' einer der Besitzer die Graberlaubnis verweigerte. Einmal gelang es jedoch Prof. Wille rom Historischen Verein Heilbronn und mir, eine grössere Grundfläche freizulegen und bis auf den Naturboden auszugraben. Das Ergebnis war eine mächtige, an den Rändern allmählich ausgehende schwarze Küulturschicht, durchsetzt mit einem regellosen Durcheinander grösserer und kleinerer Gruben mit tiefschwarzer, scherbenarmer Erde. Ein kleinerer, mitteninne liegender Komplex erwies sich durch einen grossen Mahlstein und Fenerspuren als einer Wohnstätte angehörend; cr licss sich aber nicht zu einer bestimmten Hüttenform abgrenzen. Hütteubewurf war keiner rorhanden, dagegen fanden sich durch die ganze Kulturschicht Einzelscherben rom Grossgartacher 'Typus, am wenigsten in der Tiefe der grossen Gruben, die meist mit speckiger, schollig brechender, tiefschwarzer Erde erfïllt waren. Diese vielen Gruben sind schwerlich mit einem Malc entstanden. Sie gehörten auch ihrer ganzen Anordnung nach sichtlich nicht etזra nahe aneinanderstehenden Hütten an. Wir hatten anfangs an Viehstände geglaubt, die den Boden bis in grosse Tiefe mit Jauche durchtränkt und rerändert bätten; nach den Erfahrungen am Main ist jedoch eine andere Erklärungsweise nahrscheinlich: Der ganze grosse Kulturplatr mit seiner allmählich ausgehendeu Schicht von Kulturerde ist sicher als grosse Tiehhürde anzusehen, in welcher die Hirten bei der Bewützung leichte Hütten mit bodenständigem Dach ohne Steilwände errichteten. Er diente als Winterquartier für die während des Sommers in den Wiesengründen weidenden Herden. Das Dach der Hütten müssen wir uns als mit Reisholz medecktes Gestänge denken, in dessen Innern zum Schutz gegen den Winter: tiefe Gruben gegraben wurden. Im Sommer zerfiel der Oberbau und die Gruben füllten sich durch Regengüsse mit Schwemmerde. Im nächsten Winter wurden, wo sich die alten Hütten nicht ausbessern liessen, neue Winrerhütten in der gleichen Weise auf dem schon vielfach benutzten Boden errichtet, die dann früherc Hüttengruben überschneiden konnten. Nenes Scherbenmaterial war dann vom alten durch eine Schwemmschicht getrennt. Beobachtet haben wir dies in Grossgartach mit seiner Gleichzeitigkeit verschiedener Stilformen jedoch noch nicht. Hier haben wir also eine ganz andere Wirtschaftsform wie bei den regelmässig gestellten Gehöften: Weidewirtschaft mit Überwintern des Tiehs im Pferch und Hirtenwohnungen mit bodenständigem Dach, zum vorübergehenden Gehrauch errichtet.

Damit ist das Bild der wirtschaftlichen Verbältnisse in der grossen Grossgartacher Niederlassung und ilırer Beziehung zu den Wohnformen rinem vorläufigen Abschluss gelangt. Wir haben dann die dortigen Ausgrabungen vorerst eingestellt, namentlicb auch, weil die Tatsache, dass wir 1911, um den Kongressteilnehmern kurz vor der Ernteycit etwas Anschanliches hieten zu können, auch für nicht im Ertrag stehende Äcker erhelb. liche Entschädigung der Graherlaubnis bewilligt hatten, die Ackerbesit:ar für vernünftige Forderungen un\%ugänglich gemasht hatte. Zu hemerken ist noch, dass die Probeuntersuchung allor. Stellen, welche schom von aussen 
durch dunklere Färbung der Oberfläche und durch den Pflug zutage gekommene Scherben kenntlich sind, ergeben hat, dass sie keinen festgebauten Wohnhäusern, sondern leichten Hütten ohne durch Lehmverputz gefestigte Wände, die verschiedenen Ackerbaubetriebszwecken dienten, entstammten.
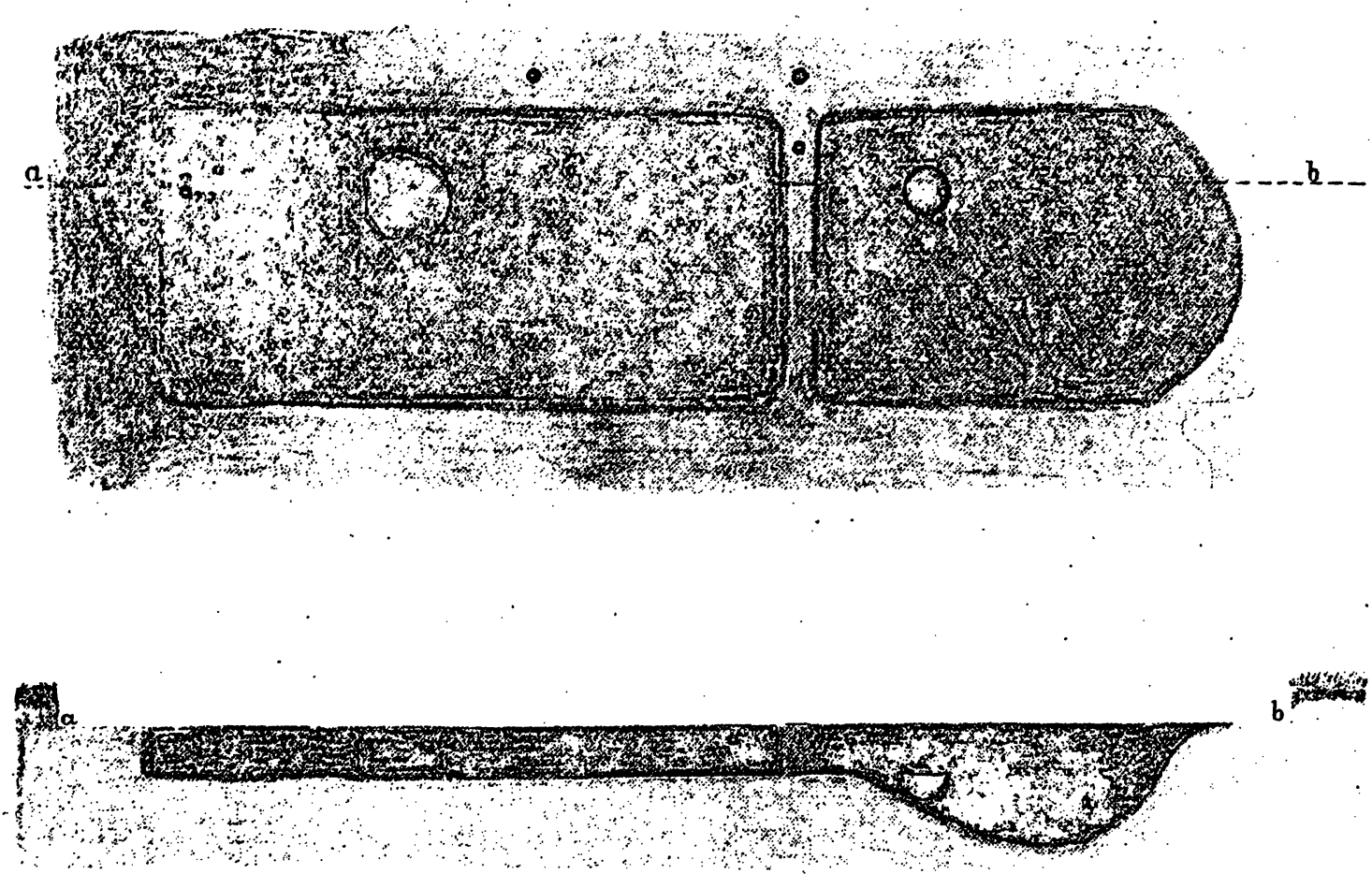

\section{HEILBRONN $\% \mathrm{~N}$}
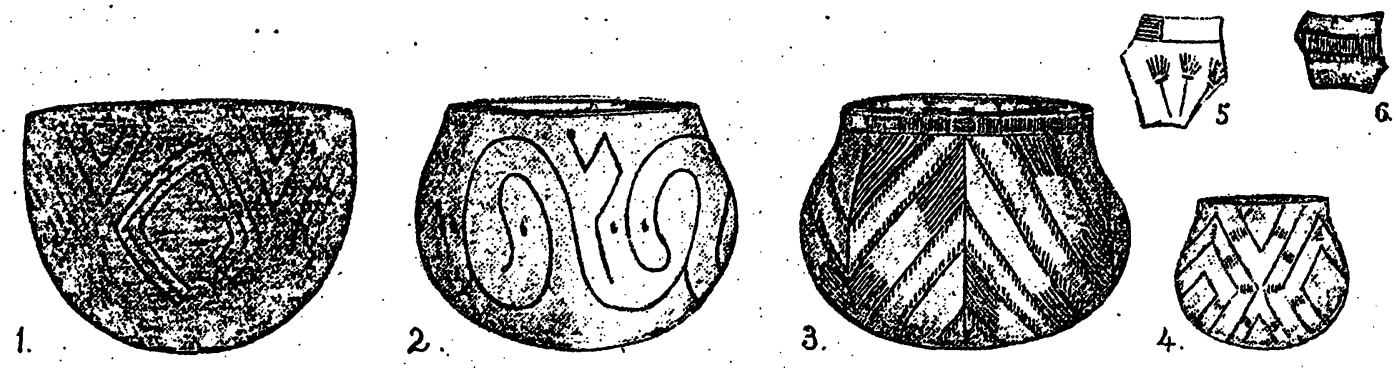

Abb. 1. Haus zwischen Heilbronn und; Neckarsulm.

Wir haben dann unsere Aufmerksamkeit dem N e ckartal zugewenlet. Neolithisch besiedelt sind dort die Hochufer, namentlich das rechte bis zum Eintritt des Flusses in den Odenwaldeinschnitt, gewesen. Hier waren schon früher bei Eisenbahnbauten südlich Neckarsulm und unterhalb sowie oberhalb Heilbronns Hüttengruben mit Scherben und Knochen zum Vor- 
schein gekommen. Sie müssen aber kleinen und schmalen Hütten angehört haben, denn der Altertumsforscher Ganzhorn in Neckarsulm hielt sie für Gräber mit Beigaben von Gefässen und Tieropfern. Die Begehung der Felder hatte seitdem keine Siedlnngsreste zutage gebracht, dagegen sind bei Tiefgrabungen eine halbe Stunde unterhalb und eine Stunde oberhalb Heilbromns Wohnstätten zum Vorschein gekommen, deren Anlage besonderes Interesse verdient.

Die eine lag $\mathrm{z} w$ ischen $\mathrm{He}$ ilbronn und Neckarsul mund war beim Baumsatz angeschnitten worden. Da der Grundbesitzer selbst Verstïnduis für die wissenschaftlichen Fragen besass, so liess ich durch ihn die ganze Stelle weit über die sichtbare Begrenzung freilegen. Sie zeigte sich nun als langes, schmales Rechteck schwarzer Kulturerde, die sich bei der tusgrabung als nur $30 \mathrm{~cm}$ stark erwies. Das Innere war eingelassig, ohne vertieften Kücheuraum und besass als einzige Einrichtung einen grossen flachen Steintisch, aber weder Kochgrube noch Vorratsgrube, sondern ganz flachen Boden. Auch der Hüttenbewurf fehlte ganz. Durch eine schmale I.ehmirand getrennt, schloss sich an der dem Eingang gegenüberliegenden Sclmalseite ein kleineres Rechteck mit bis in $\mathbf{9 0}$ cm Tiefe tiefschwarz gefärbteui Borlen an, wie wir ihn ron den Grossgartacher Stallungen her kennen. Dic Cimgebung der Gruben ergab keine Pfostenstellungen, aber mehrere $30 \mathrm{~cm}$ rom Rand entfernte senkrechte Pfahllöcher, sich rom gelben Lehm rund abhebend (Abb. 1).

Die zweite neolithische Wohnstätte wurde bei L a uf e n a $\mathrm{Neckar}$ auf der Höhe des linksufrigen „Steetsfelds“ beim Graben eines Rübenloches cntrleckt. Sie lag $70 \mathrm{~cm}$ unter der jetzigen Bodenoberfläche. Die Ausgrabung geschah durch den Historischen Verein Heilbronn unter Beisein des Leiters des Lauffener Altertumsmuseums und mehrerer Lauffener Lehrer. Den Verlauf und das Ergebnis der Ausgrabung erweist folgendes nach der Grabung aufgenommene, von den beteiligten Herren unterzeichnete Protokoll:

.Beim Eintiefen einer Rübenmiete hatte der Grundbesitzer Gottlob Beyle in Lauffen a. N. eine Anzahl linienverzierter Gefässbruchstücke, in tiefschwarzer Erde liegend, zutage gefördert, welche zweifellos neolithischen Ersprungs raren. Auf Anruf des Museumsleiters von Lauffen, Herrn Th. Schlayer, dem noch weitere Scherben von anderen Äckern übergeben vorden waren, unternahm der Historische Verein Heilbronn am 13. März 1914 die Intersuchung dieser Stellen. Anwesend waren die Herren Prof. Dr. Friz, Silnriftuihrer, O. Kress, Rechner, Ir. Schliz, Vorstand des Heilhronner Verrins, und Th. Schlayer, Iseiter des I tauffener Museums. Gegralen wurde rurch den Ackerbesitzer G. Beyle, die Besitzer des Nachbarackers, Brüder Fherbach, und einen Tagelöhner. Da die Stelle des Fundes der Scherben genau bekannt war, wurde znnächst ein Probegraben von $10 \mathrm{~m}$ Länge und $1 \mathrm{~m}$ Breite durch diese Stelle bis auf den Beginn des schwarzen Bodens gelegt. Nach der Ausschachtung der Ackererde bis in $70 \mathrm{~cm}$ Tiefe zeigte sich anf der Soble des Grabens eine gleichmässige Fläche tintenschwarzen Bodens in einer Länge ron $3,50 \mathrm{~m}$. Dabei stellte sich heraus, dass der Graben 
den südlichen Rand der schwarzen Erde freigelegt hatte, welcher sich in geradliniger Fincht gegen den umgebenden gelben Lössgrund abhob. Rechtwinklig zur Mitte des ersten wurde ein zweiter Graben von gleicher Breite

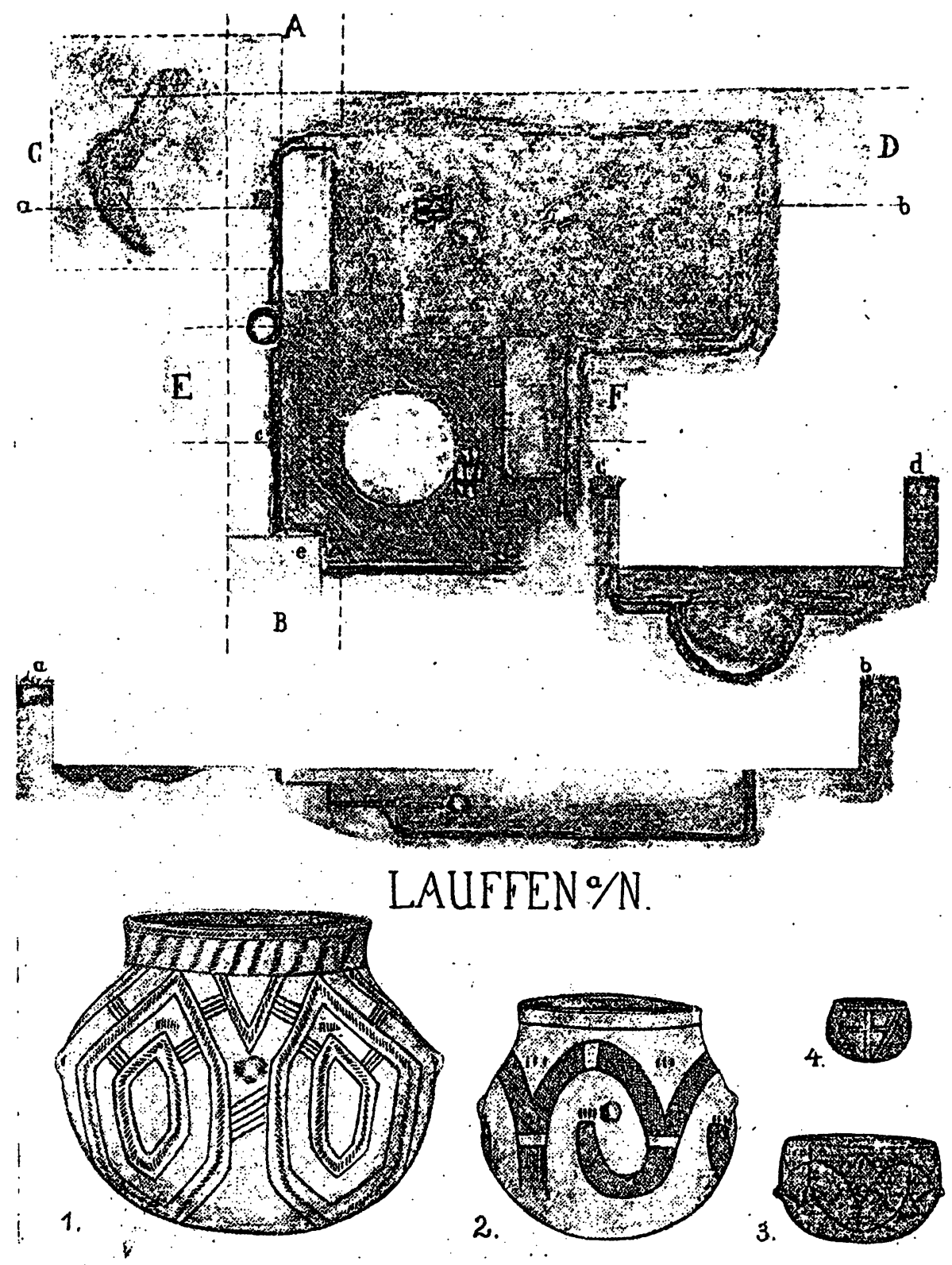

Abb. 2. Haus bei Lauffen a. N.

und Tiefe angelegt, in welchem die tiefschwarze Erde in einer Ausdehnung von 2,30 $\mathrm{m}$ als dortige Breite der Wohngrube zum Vorschein kam. Um den westlichen Rand der schwarzen Schicht freizulegen, legten wir da, wo sie im ersten Graben ihr Ende fand, einen dritten gleichen Graben ron $10 \mathrm{~m}$ Länge, ebenfalls rechtwinklig zum ersten, an, welcher auf seinem Grund 
anch hier wieler den Rand der schwarzen Schicht geradlinig in sich fasste. Hier ergab die Breitenausdehnung der Wolngrube $4 \mathrm{~m}$, also 1,70 $\mathrm{m}$ mehr als beim zweiten Graben. Nun legten wir die gesamte schwarzeFläche, anfangend an der ursprünglichen Fundstelle, in ihrer ganzen Ausdehnung frei. Die Form derselben ergab zwei rechtwinklig aneinander stossende Rechtecke, ein östliches von $2: 2,30 m$ Flächeninhalt und ein westliches von $4 m$ Länge und 1,60 m Breite. Diese geringe Breite schien uns die Verlängerung des westlichen Teils über den mittleren Breitendurchmesser hinaus als Anbau zu charakterisieren. Wir. vermuteten daher anfangs hier den Eingang. Eingefasst war der gesante rechteckige Umriss, der sich clurch die tiefschwarze, beim Abschälen glänzend erscheinende Erde des Inuerṇ besonders scharf gegen die Umgebung abhob, ron einem gleichmässig $20 \mathrm{~cm}$ breiten Streifen rötlich-brauner Erde ohne Einschlüsse, an wclchen sich erst der gelbe Jöss anschloss. Die Grube war sichtlich lange Zeit mit Regenwasser gefüllt gewesen, so dass sich die Oberfläche des Innern mit schwarzem Schlick üherzog und der an die Ränder anstossende Naturboden sich mit Kulturlange imprägnierte. Die Aushebung der schwarzen Erde bis anf den Naturboden ergab folgendes Bild: Das Rübenloch hatte nur die südwestliche Ecke der Grube in einer Ausdehnung von 50:60 $\mathrm{cm}$ geschnitten; an der gegenüberliegenden nordöstlichen Ecke bestand eine Abschrägung ron $60 \mathrm{~cm}$ Breite. In diesem Rechteck war die Kulturschicht nur $30 \mathrm{~cm}$ stark, in der Mitte zeigte sich jedoch eine kreisrunde Grube von $1 \mathrm{~m}$ Durchmesser und $60 \mathrm{~cm}$ 'Ticfe, die sich durch verschlackte Steine und vereinzelte Lehmbrocken als Kochgrube auswies. Neben ihr verlief eine Lehmbank von $25 \mathrm{~cm}$ Höhe mnd $50 \mathrm{~cm}$ Breite am östlichen Rand. Eine gleiche, $40 \mathrm{~cm}$ breite Lehmbank schloss sich in eiuer Länge von $1,20 \mathrm{~m}$ an die westliche Seite an. Neben der Kochgrube war eine Pflasterung aus in der Mitte gespaltenen grossen Rollsteinen angelegt. Sonst war der Boden gleichmässig ebeu. Weit mächtiger (60 $\mathrm{cm}$ stark) war die Kulturschicht im anstossenden rechteckigen Anbau. Nach der Ausgrabung zeigte sich hier der Boden gleichmässig $1 \mathrm{~m} 30 \mathrm{~cm}$ gregen den übrigen Hüttenboden vertieft. Auch hier fand sich eine Steinpflasterung, daneben ein ganz erhaltenes bombenförmiges Gefäss. Als Kulturreste ergab die Ausgrabung sparsam Tierknochen, einige Fenersteinartefakte und reichlich Gefässbruchstücke, die grösstenteils verzicrt waren. Fis fand sich lediglich kein Hüttenbewurf, die Wände waren sicher nicht wit Lehmrerputz gefestigt gewesen; die Grubenwände waren jerloch senkrecht abgestochen, sonst hätte sie das stagnirrende Wasser nicht in so gleichnüissiger Schicht verfärben können. Wir hatten also eine rechteckige neolithische Hüktengrube mit einem rechteckigen Anbau vor uns, der vertieft und mit Stren ausgelegt als Schlafraum diente. Der östliche, flachere Teil mit Korhgrube und Lehmlänken diente als Kïche. Der Eingung war an rer alygosclirägten Ecke, denn hier erwies sich die Tingehung durch in den Bulen ejngetretenen Abfall noch eine flache Sitrecke verfärbl."

Lauffen, 13. März 1914.

J)r. Friz. Otto Kress. A. Schliz. Th. Schlayer. 
Bei der Anlage des zweiten Quergrabens hatte sich $2 m$ vom Rand der Hüttengrube entfernt wieder schwarze, wenn auch heller gefärbte Ercle mit vereinzelten Scherben gezeigt. Da wir hier ein Vorratshaus vermuteten. und wir die Frage des Bestehens von Pfosten- oder Pfahlstellungen in weiterer Entfernung von den Rändern der Grube nochmals von einem Sachverständigen prüfen lassen wollten, habe ich eine zweite Grabung mit G. B e r s u und P. Gö s s le r veranlasst. Pfosten- oder Pfahlspuren wurden auch diesmal nicht gefunden, dagegen zeigte sich, dass die zweite, Wohnspuren aufweisende Stelle aus einer unregelmässig begrenzten Schicht vereinzelte Tonscherben enthaltender dunkler Kulturerde bestand, die sich in einzelnen Flecken auch in der Umgebung noch vorfand. Der Grund der Stelle ergab eine Reihe tieferer und flacherer, auf ihren Zweck unbestimmbarer Gruben und Gräbchen. Wir kamen gemeinsam zu der Ansicht, dass hier kein Gebäude stand, sondern dass wir den n e olith is che $\mathrm{n} \mathrm{H} \mathrm{u} \mathrm{m} \mathrm{u} \mathrm{s}$ innerhalb eines sich an das Wohnhaus anschliessenden Hofes vor uns hatten, der vielleicht für Hausviehhaltung oder. Hackbaubeete diente. Ein besonderes Interesse bekommt diese Beobachtung dadurch, dass das damalige Niveau der Hüttenumgebung der Randhöhe der Wolnngrube entsprach, dass also diese damals nur $30 \mathrm{bzw} .60 \mathrm{~cm}$ in die Bodenoberfläche eingeschnitten war: Die $70 \mathrm{~cm}$ hohe Lössbedeckung der Jetztzeit, die die ganze Anlage bewahrt hat, entstammt also der bekannten Bodenwanderung des Löss. Weiter fand sich bei dieser Ergänzungsgrabung ausserhalb der Hüttenbegrenzung unweit des südlichen Randes der Grube im scheinbar unberührten Löss ein Scherbennest, aus dessen Stücken sich ein grosses, linear verziertes, amphorenartiges Gefäss zusammenstellen liess. Es stand offen bar zur neolithischen Zeit in einer vom Innern aus in die Wand gegrabenen Nische, wahrscheinlich als Wasserbehälter dienend. Wir haben dieses Vorkommnis auch in Grossgartach dreimal angetroffen.

Diese beiden Wohnanlagen auf den Hochufern des Neckars unterscheiden sich in wesentlichen Punkten von denen der vom Lauffener Haus doch nur etwa eine Stunde Wegss entfernten Grossgartacher Niederlassung. Auch hier haben wir rechteckige Grundrisse, aber die Häuser sind weit weniger tief in den Boden eingeschnitten; es fehlt das vertiefte Küchen- oder Kellergeschoss und jeder Hüttenbewurf. Es fehlen gesonderte Stall- und Vorratsgebäude. Bezeichnend ist als diesen beiden Neckarwohnstätten gemeinsam Steintisch und Steinpflaster im Innern. Auch die hier gefundene $\mathrm{K}$ e r a $\mathrm{mik}$ unterscheidet sich in wesentlichen Punkten von der Grossgartacher. Von der L a u f e n e r Hütte haben wir vier Gefässe abgebildet. Nr. 1 und 2 sind mainländische Typen. Die gebrochenen Freiornamente von Nr. 1 kennen wir von Heldenbergen bei Friedberg, ebenfalls als Amphorendekoration, und die Schlangenbänder von Nr. 2 mit ihren gekreuzten Strichlagen sind Eichelsbacher Typus, vom Main über den Mittelrhein bis nach Belgien (La Hesbaye) verbreitet. Beide Dekorationen fehlen in Grossgartach. Die streng geometrisch in die Gefässwand eingeteilten Arkaden von Nr. 3 und die geraden Stichlinien von Nr. 4 sind frühe Regensburger Typen. Aus der Heil b r o n n e r Hütte dagegen sehen wir in Nr. 1 und 2 Typen der: 
Flomborner Spiralmiaandermanier, $\mathrm{Nr} 3$ stellt sich als Regensburger Hinkelsteingefäss des alten Typus der oberen Donau dar. Die Winkelbäuder von Nr. 4 und das Palmblattornament von Nr. 5 sind Grossgartach eigen, und Nr. 6 ist endlich ein Randstück eines schwarztonigen polierten Gefässes voui Grossgartacher weissgefüllten Stichreihentypus. Wir haben also auch hier wieder spät und früh angesetzte Formen, winklige und gebogene Linearbandornamente mit Stichbandtypus in denselben vollkommen isoliert gelesenen Einzelwohnstätten vereinigt. Nur die zweifellos den Schluss der bandkeramischen Stilbewegung darstellenden gemalten Bandornamente fehlen. WerLusthat, kannja aus diesen Funden wieder eine neue Chronologie a f fauen.

Und nun die $W^{\gamma}$ i t $\mathrm{seb}$ a f $\mathrm{t}$ f orm, der diese Einzelhütten dienten. Bei der Heilbronner Wolınstiitte haben wir der Einfachlieit ihres Grundrisses und ihrer Einrichtung wegen an eine Hirtenwohnung für die Sommerweide gedacht; die Lauffener Wohnstätte ist aber doch schon ein recht wohl eingerichtetes, mit architektonischem Bedacht konstruiertes Wohnhaus. In Grossgartach haben wir regelmässige Ackerbaugehöfte inmitten des zum Pflugbau geeigneten Geländes. Der feste Wohnsitz verlangt Wohmhaus, Keller, Speicher und Stall. Es fehlt dort daher auch jede UUberschneidung der Hïttengruben, wie sie nur bei einigermassen fluktnierender Bevölkerung; die in den weiten Flusstälern mit ihren Herclen auf Sommerweide zog und in Winter nach den alten, meist durch die Lage der Quellen bestimmten Wohnplätzen zurückkehrte, denkbar ist. Hier am Neckarhochufer sehen wir dagegen Einzelhütten, einfach aber stabil gebaut, mit einem grossen Reichtum keramischer Ware sowohl vom Main-Rheingebiet, als von der Donau her. Wir werden nicht fehlgehen, wenn wir diese über dem Flussufer angelegten Einzelanlagen als $S \mathrm{ch}$ if f a h r t s - und Handelss t a $t$ i o n e n mit nur dem Eigenbedarf dienenden Pflanzenbau- und Haustierzuchtbetrieb ansehen. Wir haben damit innerhalb der bandkeramischen Tollkultur drei verschiedene Wirtschaftsformen.

Line weitere Frage ist aber noch der Lösung näherzubringen, nämlich wie es kommt, dass die Rechteckform der Hütten bei der geringen Tiefe des in den Boden eingeschnittenen Innern sich so scliarf erhalten hat, trot\%dem hier lehmverputzte Flechtwerkwände, wie in Grossgartach, sichtlich nicht vorhanden waren. Bei den Grossgartacher Wohnhäusern, deren flechtwerkgefüllten: aus Rundhölzern gehildeten Rahmenbau wir ans den Abdriicken in len grosesn Wandrerputzstücken obne weiteres rekonstruieren kömmen. ist es leicht verständlich, dass die Ränder der Gruben in der ursprïng]iclıen Form erhalten geblieben sind; bei den Neckartalhäusern muss der Raull in irgendeiner anderen Weise gefestigt gewesen sein. Bei der geringen Brejte dieser langen Rechtecke ist las auf dem Boden aufruhende Zeltdach olıne Steilmände ausgeschlossen; es hätte das aufrechte Stehen im Innern, in lem doch, wie aus dem Steintisch und dem Kulturinhalt hervorgeht, alle mögliche Hantierung getrieben wurde, nicht erlaubt. Wir müssen also don O be $r$ b a u der Hütte nach rorhandenen Analogien in möglichst einleuch. tender Weise rekonstruieren. 
A. Schliz,
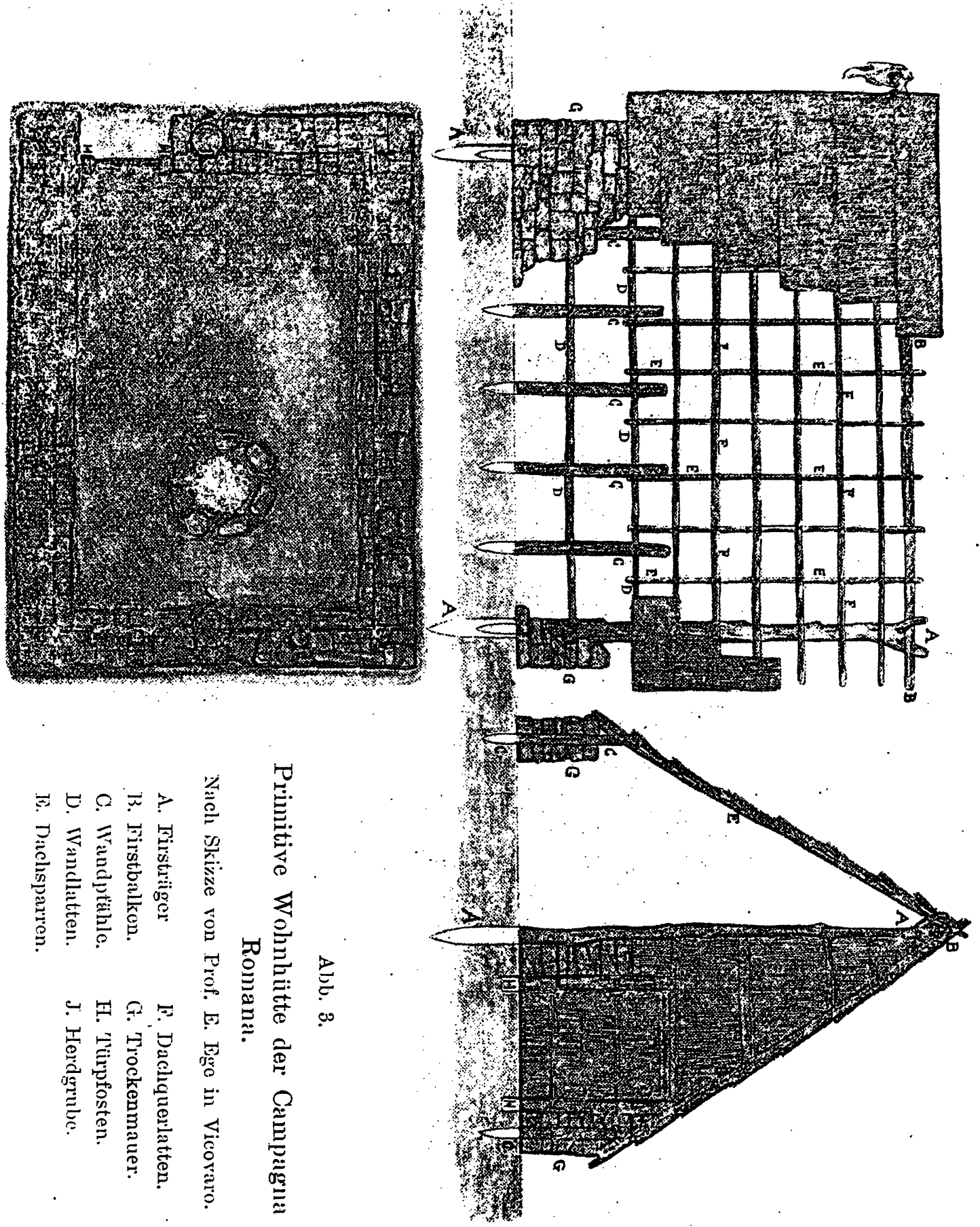
Iis sind mir nun bei einer Reise durch Latium nach Rom die zahlreichen, meist rechteckigen, primitiven Hütten aus mit Rohr odcr Reisholz verkleidetem Pfahlwerk, die einzeln im Felde stehen, aufgefallen. Professor. Eberhard Exe, Ispettore onorario dej Monumenti e Scavi in Vicoraro, mit dem ich einige dieser Hütten besuchte, versicherte mir, dass sie auch heute noch nicht nur als Ställe und Speicher, sondern auch als Wohnungen benützt würden. Ich bat ilın, sich ïl)er die Konstruktion des Aufbaues an zuständiger Stelle eingehend zu unterrichten und mir einige Photogramme typischer Hütten zu besorgen. Er schickte mir eine Zeichnung, die in Abb. 3 wiedergegeben ist, und schreibt dazu:

.Ich erlanbe mir, Ihuen dic genaue Beschreibung der Konstruktion der: Hütten zu schicken, über clie ich mich hei se! m zuverlässigen Personen inforniert habe. Die Hiitten werden durchweg nach dem gleichen Prinzip konstruiert, sowohl die runden wie die viereckigen, und zwar folgendermassen: Znerst werden icẹi ziemlich starke Stangen A im Boden befestigt. Dic Stangen haben oben natürliche Gabelung, manchunal wird auch einfach ein gerrachscner Baum stehen gelassen und abgeschnitten, um als eine der Stangen A zu dienen. Hierauf wird die Querstange B befestigt, dann werden dem Umfang, den die Ḧ̈̈tte bekommen soll, entsprechend die Pfähle C eiugerammt und durch die Querlatten $D$ rerbunden. L's erfolgt sodann die Befestigung der Stangen $\mathrm{E}$, welche durch die Querlatten $\mathrm{F}$ verbunden werclen. Ist so das Holzgerüst fertiggestellt, so wird die Mauer G olne Mörtel von Bruchsteinen gelegt, und zwair so, dass die Hölzer C, D die Mitte der Mauer traversieren, also gleichsam das Skelett der Mauer bilden. Ist die Hütte etwas luxuriöser, so werden die äusseren Fugen der Mauer mit Lehm oder Mörtel ausgekittet, gewöhnlich aber bleibt sie ohne Verkittung. Die Tür liegt cntweler an der Vorderseite oder an der Langseite, aber niemals in der Vitte, immer gegen das Eck zu. . Da die Tür höher ist als die gelegrte Maner, so wird zu beiden Seiten der Tür die Mauer höher aufgefübrt, manchmal auch richtig gemauert mit Mörtel; die ältere Konstrulition ist aher jedenfalls die. dass zu beiden Seiten der Tür etwas hölıere Pfähle H eingerammt werden, an denen die Tür befestigt wird. Statt der gelegten Mauer G rird häufig das senkrechte Gerüst C, D einfach mit Stroh bekleidet nder mit $\mathrm{Z}$ weigen mit Laub daran. Manchmal wird die Hütte mit einem kleinen Grabeu umzogen, damit das Wasser abläuft. Der Steinsockel dicut also nicht als Widerlager; die Festigkeit liegt in clem Holzgerüst. Bei ganz einfarhen Hiitten gehen die Stangen $\mathrm{E}$ bis zur Errle, und fällt die senkrechte $T$ and $G$ weg. Die Mauer $G$ ist mindestens $1 \mathrm{~m}$ stark und ungefähr 1,20 m hoch. lis konmt vor, dass arme IJeute gan\% in diesen Hütten wohnen, ist aly: $r$ selten; meistens wohnen die Bauern in diesen Hütten den Sommer über in der Kampagna, hesonders zur Zeit der Erute. Ijchtöffnungen sind selten angebracht; wenn eine solche angebracht ist, befindet sie sjch an der Giebelwand. Ansser zu Wohnzwecken dienen diese Hütten als Speicher und als Ställe, kleine als Schweine- oder Hühnerställe. An den Gjebelı sind geWöhnlich Pferde- orler Haultierschählol angebracht gegen den hïs(en Plirek." 
Um aus dieser Bauweise eine Nutzanwendung auf den Oberbau unserer nenlithischen Häuser zu ziehen, ist auf die ausserhalb der Ränder der Heilbronner Hütte in gleichmässigem Abstand von $30 \mathrm{~cm}$ gefundenen senkrechten Pfabllöcher zurückzukommen. Auch bei einer gemeinsam mit K. Schumacher und P. Gössler vorgenommenen Ausgrabung eines Stadels im Aussenbezirk der Grossgartacher Niederlassung fanden sich, wie ich in meinem Vortrage auf der Weimarer Tagung ${ }^{1}$ ) erwähnte, in Reihen stehende kreisrunde Stellen lockcrer, nicht dern Naturboden entsprechendèr Erde ausserhalb der Hüttengrubenbegrenzung. Da zur Zeit dieser Grabung Regenwetter herrschte, traten sie auf dem nassen Boden nicht deutlich hervor, als ich jedoch die Stelle später nochmals bei trockenem Wetter in ihrer ganzen Ausdelınung freilegte, erschienen sie deutlich als dunklere, in den Boden sich senkrecht eintiefende, einem schwachen Pfahldurchmesser entsprechende Kreise .veränderter Erde. Wir sind später nicht mehr auf diese Grabung zurückgekommen, weil es sich bei derselben um die Form des Hüttenumrisses handelte und eine solche deshalb nicht bestimmt festgestellt werden konnte, weil unter der oberen leichten Vorratshütte diagonal das Untergeschoss eines früher hier gestandenen abgebrannten Hauses lag, von dessen Aussengrenzen Teile ausserhalb der zweiten Anlage zum Vorschein kamen.

Übertragen wir nun die Banweise der Wände der Campagnahü̈ten auf unsere neolithischen Bauten, so erhalten wir einen Oberbau aus einem in den Naturboden eingesteckten Stangengerüst, das dann durch einen Mantel schweren, festen Materials Halt und Fèstigkeit bekam. Bei dem vollständigen Fehlen lagerhafter Bruchsteine im Löss wurde die Verstärkungsmauer aus luftgetrockneten Lehmziegel n erbaut, deren Kenntnis wir bei dem hohen Stand der Töpferkunst in dieser Kultur ohne weiteres annehmen können. Die in der Mauer eingeschlossenen, keiner Imprägnierung durch Kulturlauge ausgesetzt gewesenen Pfähle sind grossenteils zergangen, ohne sichtbare Spuren zu hinterlassen, und die Lehmmauer zerfloss nach Zerfall des schützenden Daches und deckte die Ränder mit einer sicl vom Naturboden nicht unterscheidenden Erde zu.

Es erübrigt noch, nachzusehen, was wir von einer ähnlichen Baureise aus neolithischer Zeit aus anderen Gegenden wissen. Wir ziehen zu diesem Zweck Ed. Me y ex, „G e s c hl i c h t e d e s A l t e r tu m s I, 2 1913“ heran, ein Buch, von dem wir annehmen können, dass es dem jetzigen Stand unserer Wissenschaft in diesen Dingen entspricht.

Zunächst sagt er über das R e c h t e ck ha u s, $\oint 509$, Thes s a li e n betreffend: „Neben den alten Rundhütten hat seit frühneolithischer Zeit das rechteckige Wohnhaus gestanden, ursprünglich eine einfache Wolnnkammer mit Lehmwänden und Eckpfosten, die die Dachbalken trugen, dann durch Verbindung mehrerer Kammern zu. einem wirklichen Hause erweitert. In Orchomenos finden wir Herd- und Abfallgruben, wie in den neolithischen Häusern Deutschlands. Hier wie sonst ist es falsch,

1) Ausgrabungsproblene, Korrespondenzbl. der Deutschen anthropol. Gesellschaltt. 1912. 
eine Form für die alleinige und ursprüngliche zu erklären.“

$\$ 527$, P e l o p o n n e s betreffend: „Schon in der Zeit der Steinzeitkultur sind Griechen dorthin gekommen, und die Bewohner von Orchomenos werden in der Zeit, als eine Ortschaft mit rechteckigen Häusern angeleg't wurde, bereits Griechen gewesen sein."

$\$ 511$. Ph ylakopi (Melos), woher die Obsidiammesser bezogen wurden, betreffend. „In ältester Zeit (Mitte des 3. Jahrtausends) bestand planmässige Anlage durchweg mit rechteckigen Häusern.“"

\$493/97. In der zweiten, ältestbronzezeitlichen Ansiedlung von Troja findet sich im Herrenhaus ,ein grosser Saal mit dem Herd in der Mitte und einer Vorhalle, daneben kleinere Gebäude mit durchweg rechteckigen Zimmern. Die lineare Dekoration der ältesten Zeit auf den Gefässen hat sich in rohe Nachbildung von Bäumen und Gehängen verwandelt. Es besteht $V$ e $r$ bindung mit dem Norden, wit den Fundender jüngeren neolithischen Zeit aus Mittel-und Osteuropa, ror allem ausdem Donaugebiet, einsich kreuzender Austausch."

Hier findet sich mun folgender $\mathrm{H}$ a u s b a u: „Mauer aus grossen, rechteckigen Iuftziegeln, zwischen denen Holzbalken eingelegt waren. Alle Bauten wurden aus grossen Luftziegeln aufgeführt, in die Wände sind Längsbalken eingelassen, die Dachbalken ruhen auf den Wänden." Auch in der chetitischen Kultur (Gebäude von Bogasköi) findet sich diese Bauweise mit „Holzfachwerk und ungebrannten Lehmziegeln“. Die solideren auf Bruchsteinfundament sind erhalten geblieben, „die einfacheren werden zerflossen sein".

Wir sehen also hier, wo speziell Einfluss aus der jüngeren neolithischen Zeit aus dem Gebiet der Donaukultur angenommen wird, dieselbe Wandkoustruktion neolithischer Häuser, wie wir sie für unsere Neckartalhäuser angenommen haben.

Wir schliessen mit dem Satz aus $\$ 563$ : „Schon in der Einheitszeit der Indogermanen sind die Lebensverhältnisse der einzelnen Stämme vicht gleichartig gewesen; es hat neben fortgeschrittenen, zur Sesshaftigkeit gelangten Stämmen auch nomadisierende, wesentlich oder ausschliesslich vou Viehzucht und Jagd lebende gegeben." Daraus ist $\mathrm{zu}$ ersehen, dass die Wirtschaftsform unserer neolithischen Zeit eine recht vielgestaltige war und lass wir aus den Verbältnissen in einem kleinen Teilgebiet njcht auf unser ranzes neolithisches Gebiet Schlüsse ziehen dürfen. 